\title{
PRODUTIVIDADE DE CORDEIRAS CORRIEDALE FENOTIPICAMENTE RESISTENTES A NEMATOIDES GASTRINTESTINAIS
}

${ }^{1}$ Mestrando do Programa de Pós-Graduação em Zootecnia, Universidade Federal de Pelotas; ${ }^{2}$ Professora Adjunta da Universidade Federal de Santa Maria, Campus Palmeira das Missões; ${ }^{3}$ Residente na área de Doenças

e Zoonoses Parasitárias, Universidade Federal de Pelotas; ${ }^{4}$ Doutorando do Programa de Pós-Graduação em Zootecnia, Universidade Federal de Pelotas; ${ }^{5}$ Professor Adjunto do Departamento de Zootecnia da Faculdade de Agronomia Eliseu Maciel, Universidade Federal de Pelotas.

\section{RESUMO}

A busca por ovinos resistentes à ação dos nematoides é uma tendência dentro dos sistemas produtivos, porém deve-se levar em consideração a produtividade, pois de nada vale a seleção de animais resistentes à ação parasitária, se eles não atingirem os parâmetros produtivos que o mercado exige. Assim, o objetivo deste trabalho foi quantificar as características produtivas referentes ao ganho de peso, crescimento dos componentes corporais, acabamento, conformação e qualidade da lã, relacionando-as à contagem de ovos por grama de fezes (OPG), caracterizando uma provável expressão individual de resistência a esses nematoides ao longo de um ano de vida. O experimento foi conduzido no município de Herval (RS). Foram utilizadas 32 cordeiras da raça Corriedale, sendo avaliadas dos três aos 12 meses de idade. Ao final do experimento foram classificadas em dois grupos, infeç̧ão baixa (OPG médio de cada animal abaixo de 900 ao longo do experimento) e infecção elevada (OPG médio de cada animal acima de 1000 ao longo do experimento). À exceção do parâmetro crescimento em comprimento corporal $(p<0,05)$, para as demais variáveis estudadas não foram verificadas diferenças significativas entre animais com diferentes níveis de infeç̧ão parasitária.

Palavras-chave: Ovinos. Índices produtivos. Verminose. 


\section{INTRODUÇÃO}

A verminose é considerada o maior e o mais grave problema sanitário da criação de ovinos, podendo inviabilizar economicamente a atividade (BUZZULINI et al., 2007). Problema agravado constantemente pela pressão de seleção exercida pelos fármacos (vermífugos), encontrados no mercado, sobre uma população de helmintos que já apresenta alta resistência à grande maioria dos fármacos anti-helmínticos.

Segundo Silveira et al. (2013), a alta densidade de animais por área, o uso indiscriminado de anti-helmínticos e, principalmente, a relutância dos produtores em procurar orientação técnica, são alguns dos principais fatores implicados na resistência parasitaria aos tratamentos anti-helmínticos.

Algumas características fisiológicas como idade e gestação possuem o potencial de aumentar a vulnerabilidade dos animais às parasitoses. Bueno et al. (2005) e Costa et al. (2011) classificam ovinos jovens e gestantes como as categorias mais suscetíveis à verminose. Entretanto, os ovinos possuem a habilidade de adquirirem e expressarem imunidade contra os nematoides gastrintestinais, a qual é controlada geneticamente e varia substancialmente entre as diferentes raças, bem como entre os indivíduos de uma mesma raça (STEAR; MURRAY, 1994).

Nos primórdios, as raças de ovinos e caprinos evoluíram não apenas como resultado da seleção natural imposta pelas condições ambientais, mas também como resultado dos processos de seleção realizados pelo homem. Raças inglesas desenvolveram aptidão para suportar baixas temperaturas, enquanto que raças originárias dos trópicos se adaptaram em temperaturas elevadas. Em países com clima temperado, o principal nematoide parasita de ovinos é a Teladorsagia (Ostertagia) circumcincta (BENAVIDES, 2009; BISHOP; STEAR, 1999), enquanto que nos países de clima tropical destaca-se o Haemonchus contortus (AMARANTE et al., 2004). No estado do Rio Grande do Sul, caracterizado pelo clima subtropical, os parasitas de maior importância são: $H$. contortus, Trichostrongylus axei, $T$. columbriformis, $T$. circumcincta e Oesophagostomum colubianum (Amarante, 2009).

Em raças resistentes, o desenvolvimento da imunidade contra os nematoides gastrintestinais se manifesta precocemente. Porém, essas raças, ditas resistentes, apresentam algumas 
características relacionadas à produção consideradas inferiores, como ganho de peso, produção de lã, peso ao nascimento, peso ao desmame, etc. Entretanto, mesmo raças de alta produtividade e que são consideradas suscetíveis à verminose, apresentam variabilidade genética em relação à resposta imunológica contra os parasitas (BENAVIDES, 2008). Segundo Benavides (2009), há uma baixa variação de resistência dentro de linhagens ovinas, com isso é possível melhorar essa característica a parasitas gastrintestinais pela seleção em rebanhos que possuem essa variabilidade, aliando características produtivas e de resistência. Amarante (2004), observou que $100 \%$ dos animais de um rebanho ovino podem ser considerados suscetíveis nas primeiras semanas de vida, porém, à medida que vão crescendo e amadurecendo poderão desenvolver imunidade de tal forma que na idade adulta muitos deles apresentarão resistência.

A imunidade contra os nematoides adultos em ruminantes pode se manifestar pela expulsão da população adulta dos vermes, por alterações na morfologia dos parasitas e pela redução na fecundidade das fêmeas. Já a resistência contra os estágios imaturos dos nematoides se manifesta pela eliminação das larvas infectantes ou pela inibição do desenvolvimento das mesmas, hipobiose (BALIC et al., 2000). A interação entre o parasita e o sistema de defesa do hospedeiro pode resultar na morte e eliminação dos vermes, o que ocorre nos animais resistentes ou, no outro extremo, na persistência dos parasitas, aparentemente intactos, o que ocorre nos animais suscetíveis. Em uma situação de resistência intermediária, a infecção pode persistir, porém com prejuízos à sobrevivência e à fecundidade dos nematoides. $A$ enfermidade clínica (verminose) manifesta-se especialmente nos animais suscetíveis do rebanho (AMARANTE, 2004).

Há diferenças na resposta imune do hospedeiro à infecção, em razão do modo de parasitismo (de mucosa versus hematófago), da região gastrintestinal que parasitam (abomaso versus duodeno) e da carga parasitária a qual os animais foram submetidos. Outros fatores que influenciam a resposta imune são: o modo de infecção (ingestão experimental de larvas infectantes de determinado parasita versus desafio natural com várias espécies de parasitas), idade ao desafio, época do desafio, raça, condições dos variados sistemas de produção, etc. (BENAVIDES, 2009). 
A metodologia de menor custo e melhor exequibilidade na seleção para resistência é a contagem de ovos por grama de fezes (OPG) em animais jovens expostos a desafio, uma vez que a resistência é expressa pelos indivíduos quando as condições ambientais são propícias aos parasitas. Sem essa exposição se torna impossível medir o nível de resistência aos parasitas. A contagem de OPG é uma característica de alta variabilidade, o coeficiente de variação (CV) para essa medida sem transformações logarítmicas excede os $100 \%$, e possui uma estimativa de herdabilidade média, contudo sua expressão é dependente da exposição dos animais aos parasitas por meio de desafios anuais (BENAVIDES, 2009).

O objetivo deste trabalho foi quantificar as características produtivas referentes ao ganho de peso, crescimento dos componentes corporais, acabamento, conformação e a qualidade da lã em relação à contagem de ovos por grama de fezes (OPG), assim caracterizando uma provável expressão individual de resistência a esses nematoides ao longo de um ano de vida.

\section{MATERIAL E MÉTODOS}

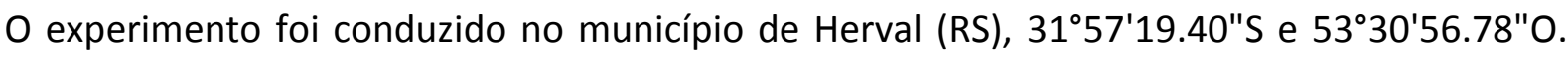
Foram utilizadas 32 cordeiras da raça Corriedale. Os animais foram avaliados dos três aos 12 meses de idade (seis avaliações), sendo everminados aos sete e aos 12 meses, logo após as avaliações, com Closantel+Albendazol e Moxidectina, respectivamente, conforme as indicações dos fabricantes. A prescrição dos anti-helmínticos objetivou evitar a possibilidade da morte de alguns animais em razão do elevado grau de parasitismo verificado após a sétima e décima segunda avaliações.

Os animais foram mantidos no mesmo potreiro e receberam o mesmo manejo alimentar até o final do experimento. Foram avaliados em relação aos seus parâmetros produtivos e a contagem de ovos de helmintos por grama de fezes (OPG). Ao final do experimento, as cordeiras foram classificadas conforme os resultados do OPG médio e ordenadas em dois grupos: infecção baixa (IB), OPG médio de cada animal abaixo de 900 ao longo do experimento; e infecção elevada (IE), OPG médio de cada animal acima de 1000 ao longo do experimento, conforme Ueno e Gonçalves (1998) para infecções mistas em animais naturalmente infectados. Após a formação dos grupos, os dados colhidos de cada animal foram utilizados para compor os parâmetros médios de cada grupo. 
Para mensurar as respostas individuais aos nematoides gastrintestinais, foram realizadas seis avaliações utilizando os dados do OPG, aos três, cinco (desmame), seis, sete, oito e 12 meses de idade, através do método de Gordon e Whitlock (1939). As variáveis analisadas para medir as características produtivas de ganho de peso, crescimento dos componentes corporais, acabamento e conformação foram, respectivamente: ganho de peso médio diário (GMD); crescimento do anterior (VAA); crescimento do posterior (VAP); crescimento em comprimento (VComp); variação no perímetro torácico (VPT); variação na compacidade (VCompa); escore de condição corporal aos 12 meses (ECC); e conformação aos 12 meses (Conf). Os valores de crescimento e variações foram dados pelas seguintes fórmulas:

GMD = (Peso final-Peso inicial) / 275 dias;

VAA = Altura final-Altura inicial;

VAP = Altura final-Altura inicial;

VComp = Comprimento final-Comprimento inicial;

VPT = Perímetro final-Perímetro inicial;

VCompa = Compacidade final-Compacidade inicial.

As variáveis de qualidade da lã foram: crescimento da mecha da lã (CL) da esquila de fevereiro (cinco meses de idade) até agosto (12 meses de idade), $\mathrm{CL}=$ comprimento finalcomprimento inicial; volume de mecha (Vol); número de ondulações por polegada (Ond/Pol); diâmetro da fibra de lã em micras (DF); variação do diâmetro da fibra ao longo da mecha durante o período de crescimento em micras (VDFM); distribuição estatística em micras que representa aproximadamente $70 \%$ das fibras que discrepam do diâmetro médio das fibras da amostra (DPF); coeficiente de variação do diâmetro (CVD); o número de micras que se afastam do diâmetro médio de $5 \%$ das fibras mais grossas em micras (MFG); porcentagem de fibras menores que 15 micras (<15); fator de conforto, ou seja, porcentagem de fibras menores ou igual a 30 micras (FC); cálculo considerando o diâmetro da fibra e o coeficiente de variação do diâmetro, representando a qualidade do fiado expressado em micras (QF); comprimento da mecha em milímetro (CM); ponto mais fino ao longo da mecha em micras (MinMic); ponto mais grosso ao longo da mecha em micras 
(MaxMic); distância da ponta até o ponto mais fino ao longo da mecha em milímetros (DistPF); e curvatura da fibra em graus por milímetro (CVR).

O número de ovos por grama de fezes (OPG) foi determinado segundo o método de Gordon e Whitlock (1939), método quantitativo que tem como princípio a flutuação de ovos de helmintos em solução saturada de cloreto de sódio, usando a câmara de McMaster, cujos dois retículos são contados ao microscópio óptico, no aumento de 100X. O peso dos ovinos foi obtido em balança tipo relógio até $50 \mathrm{~kg}$.

Conforme metodologia descrita por Osório et al. (2014), as alturas do anterior e do posterior foram obtidas pela distância compreendida entre as cruzes e o solo e entre a cabeça do fêmur e o solo, respectivamente, medidas realizadas com uma trena graduada em centímetros. O comprimento foi obtido pela medida em centímetros da distância entre as cruzes e a base da cauda. O perímetro torácico se obteve pela circunferência da cavidade torácica. A compacidade foi obtida pelo cálculo da relação do peso com o comprimento (Peso/Comprimento corporal). O escore de condição corporal foi determinado através da palpação das apófises transversas (índice de 1 a 5 , com intervalos de 0,5, sendo 1 excessivamente magra e 5 excessivamente gorda). A conformação foi determinada pelo índice de desenvolvimento corporal em uma escala de 1 a 5 com intervalos de 0,5, em que 1 é um animal muito pobre e 5 um animal excelente. Para a realização das medidas, os animais foram colocados em estação, em superfície horizontal. Para minimizar os erros inerentes ao avaliador, estas foram realizadas sempre pela mesma pessoa.

O comprimento da mecha de lã foi obtido através da medida da distância da base até sua respectiva ponta, aferido com um paquímetro graduado em milímetros, na região média do costilhar. O volume da mecha foi obtido pela mensuração do espaço tridimensional referente à multiplicação da largura, profundidade e comprimento das mechas. A Ond/Pol refere-se ao número de ondulações no mesmo plano da mesma mecha em um intervalo de 2,5 cm. As amostras de lã foram coletadas e enviadas para o laboratório Micralan, Montevidéu, Uruguai, para quantificação das medidas de DF, DPF, CVD, MFG, <15, FC, QF, CM, MinMic, MaxMic, DistPF e CVR através da utilização do Optical Fibre Diameter Analysis OFDA 2000. Todas as mensurações e amostragens foram feitas do lado esquerdo dos 
animais, uma vez que os ruminantes têm o hábito de se deitar sobre o lado oposto do rúmen, tornando o lado direito impróprio para a coleta de amostras de lã, pelas impurezas que ostenta (PINTO DE ANDRADE et al., 1999).

Para efetuar as análises estatísticas, os valores do OPG foram transformados em logaritmos na base 10, devido ao alto coeficiente de variação e para facilitar a comparação entre os grupos.

A análise estatística para os dados paramétricos de OPG e produtivos foi o teste de Fisher $(p<0,05)$ e para dados não-paramétricos (ECC, Conf e Ond/Pol) foi o teste de Kruskal-Wallis $(p<0,05)$, utilizando-se o pacote estatístico SAS 9.0.

\section{RESULTADOS E DISCUSSÃO}

Os resultados encontrados para a contagem de ovos de helmintos (OPG) estão demostrados na Tabela 1. As coletas realizadas nos meses de novembro e janeiro (prévia ao desmame) não evidenciaram diferenças significativas $(p>0,05)$, possivelmente como resultado da menor dependência das cordeiras em relação à pastagem. Fernandes (2010), Salgado (2011) e Schichowski et al. (2010), relataram que cordeiros ainda não desmamados, com uma dieta baseada no aleitamento materno, que não sofreram o estresse da desmama, são menos suscetíveis à ação dos helmintos. Ao mesmo tempo, dietas com altos teores de proteína bruta (PB) tornam os animais menos suscetíveis à verminose, nesse sentido animais em aleitamento, entre cinco e seis meses de idade, criados extensivamente, tem no leite materno 46\% da exigência de PB da dieta supridas (AMARANTE, 2009; CABRAL et al., 2008). Nas coletas subsequentes encontrou-se diferença significativa $(p<0,05)$ entre os grupos, pois os animais do grupo IB apresentaram OPGs inferiores (Tabela 1), demonstrando a menor suscetibilidade desse grupo à infecção por nematoides.

Um dos prováveis fatores da maior carga parasitária dos animais do grupo infecção elevada a partir dos seis meses de idade, em relação ao grupo infecção baixa, além da maior suscetibilidade à verminose, foi o estresse da desmama, pois conforme McClure (2000) esse manejo compromete a imunidade dos cordeiros devido ao sofrimento pela ruptura do vínculo materno-filial. Desse modo, podemos supor que o grupo IE sofreu mais intensamente esse período de transição. 
Tabela 1 - Médias das contagens de ovos por grama de fezes (OPG) de cordeiras Corriedale, em seis avaliações ao longo de um ano, no município de Herval (RS).

\begin{tabular}{|c|c|c|c|}
\hline & \multicolumn{2}{|c|}{ Infecção } & \multirow[b]{2}{*}{$\operatorname{CV}(\%)^{2}$} \\
\hline & Baixa $(n=15)^{1}$ & Elevada $(n=17)^{1}$ & \\
\hline $\begin{array}{c}\text { Novembro } 2013 \\
\text { (Três meses de idade) }\end{array}$ & 86,66 & 243,57 & 75,5 \\
\hline $\begin{array}{c}\text { Janeiro } 2014 \\
\text { (Cinco meses de idade }{ }^{3} \text { ) }\end{array}$ & 320,00 & 681,25 & 68,2 \\
\hline $\begin{array}{c}\text { Fevereiro } 2014 \\
\text { (Seis meses de idade) }\end{array}$ & $133,33^{\mathrm{a}}$ & $685,71^{b}$ & 89,0 \\
\hline $\begin{array}{c}\text { Março } 2014 \\
\text { (Sete meses de idade }{ }^{4} \text { ) }\end{array}$ & $1643,66^{\mathrm{a}}$ & $3693,33^{b}$ & 10,6 \\
\hline $\begin{array}{c}\text { Abril } 2014 \\
\text { (Oito meses de idade) }\end{array}$ & $333,33^{\mathrm{a}}$ & $750,00^{b}$ & 20,2 \\
\hline $\begin{array}{c}\text { Agosto } 2014 \\
\text { (Doze meses de idade }{ }^{4} \text { ) }\end{array}$ & $619,23^{\mathrm{a}}$ & $2273,33^{b}$ & 34,4 \\
\hline
\end{tabular}

As avaliações das características produtivas ao longo do experimento estão demonstradas na Tabela 2. Dos parâmetros avaliados, somente a variável crescimento em comprimento (Vcomp) apresentou diferença significativa $(p<0,05)$, ou seja, ovinos do grupo IB apresentaram maiores ganhos que ovinos do grupo IE. O comprimento corporal é de grande importância no rendimento da carcaça no frigorífico, ocorrendo, assim, uma melhor remuneração pelo produto (OSÓRIO; OSÓRIO, 2003). Benavides et al. (2010) e Woolaston et al. (1992) verificaram que ovinos de linhagens mais resistentes (baixo OPG) tenderam a apresentar maiores índices produtivos, principalmente maiores pesos ao nascer. 
Tabela 2 - Médias e desvios padrões das características produtivas de ganho de peso, crescimento dos componentes corporais, acabamento e conformação de cordeiras Corriedale no município de Herval (RS).

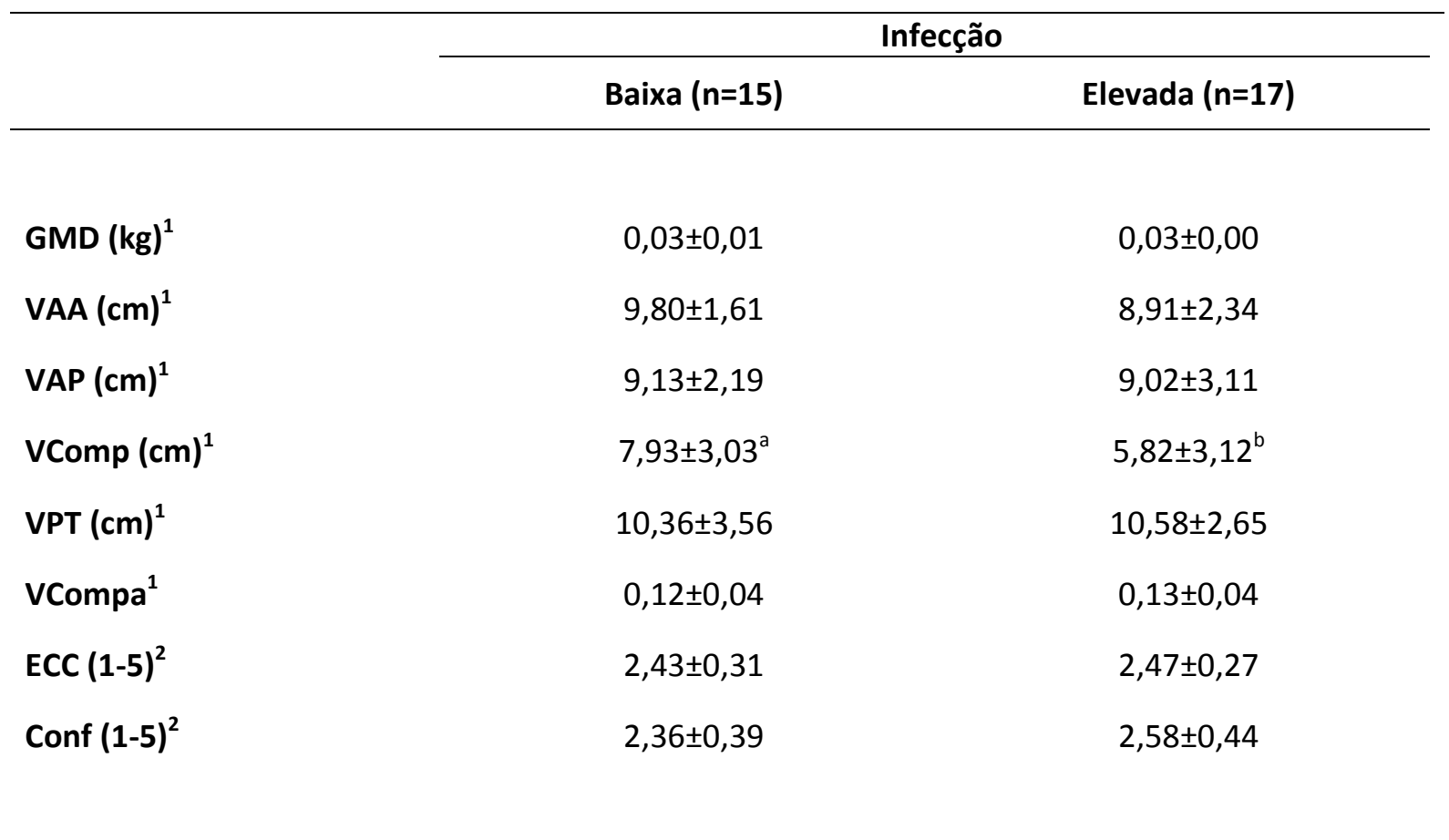

\footnotetext{
${ }^{1}$ Médias seguidas de diferentes letras na linha, diferem estatisticamente pelo teste de Fisher $(p<0,05)$.

${ }^{2}$ Médias seguidas de diferentes letras na linha, diferem estatisticamente pelo teste de Kruskal-Wallis $(p<0,05)$. $G M D=$ ganho de peso médio diário, (Peso final-Peso inicial/275 dias); VAA= crescimento do anterior, (Altura final-Altura inicial); $\mathrm{VAP}=$ crescimento do posterior, (Altura final-Altura inicial); $\mathrm{VComp}=$ crescimento em comprimento, (Comprimento final-Comprimento inicial); VPT= variação no perímetro torácico, (Perímetro finalPerímetro inicial); $\mathrm{VCompa}=$ variação na compacidade, (Compacidade final-Compacidade inicial); $\mathrm{ECC}=$ escore de condição corporal aos 12 meses; Conf= conformação aos 12 meses.
}

As características relacionadas à qualidade da lã estão demonstradas na Tabela 3. Não houve diferenças significativas $(p>0,05)$ entre os grupos para as variáveis estudadas. Resultados semelhantes foram encontrados por Benavides et al. (2010) e Woolaston et al. (1992), que não verificaram diferenças entre as características de produção de lã entre grupos de ovinos mais e menos suscetíveis à verminose (pesos de velo sujo e limpo, diâmetro médio de fibra e comprimento de mechas).

Na Austrália e Nova Zelândia, respectivamente, animais das raças Merino (EADY et al., 1998) e Romney (WILLIAMSON et al., 1995a; WILLIAMSON et al., 1995b), selecionados para características de produção de lã, apresentaram maior susceptibilidade a nematoides, provavelmente devido ao elevado nível de seleção para essa característica. Podemos inferir que pelo fato da seleção ter sido unidirecional, sem considerar características de resistência, 
os animais tenderam a perder essa característica adaptativa no decorrer dos anos de seleção.

Tabela 3 - Médias e desvios padrões das variáveis referentes à qualidade da lã de cordeiras Corriedale no município de Herval (RS).

\begin{tabular}{|c|c|c|}
\hline & & \\
\hline & Baixa $(n=15)$ & Elevada $(n=17)$ \\
\hline $\mathrm{CL}(\mathrm{cm})^{1}$ & $47,00 \pm 5,34$ & $44,88 \pm 6,42$ \\
\hline $\operatorname{Vol}\left(\mathrm{mm}^{3}\right)^{1}$ & $4967,19 \pm 1018,73$ & $4881,81 \pm 1196,92$ \\
\hline Ond/Pol ${ }^{2}$ & $6,73 \pm 1,53$ & $6,70 \pm 1,57$ \\
\hline $\mathrm{DF}(\mu)^{1}$ & $24,46 \pm 1,34$ & $24,74 \pm 1,70$ \\
\hline $\operatorname{VDFM}(\mu)^{1}$ & $-0,2 \pm 1,34$ & $0,2 \pm 1,64$ \\
\hline $\operatorname{DPF}(\mu)^{1}$ & $4,96 \pm 0,52$ & $5,22 \pm 0,90$ \\
\hline CVD (\%) ${ }^{1}$ & $20,34 \pm 2,03$ & $21,12 \pm 2,85$ \\
\hline $\operatorname{MFG}(\mu)^{1}$ & $9,29 \pm 1,12$ & $9,88 \pm 1,79$ \\
\hline$<15(\%)^{1}$ & $0,86 \pm 0,85$ & $1,01 \pm 0,85$ \\
\hline FC $(\%)^{1}$ & $87,85 \pm 5,94$ & $85,39 \pm 8,78$ \\
\hline $\mathrm{QF}(\mu)^{1}$ & $23,68 \pm 1,29$ & $24,12 \pm 1,93$ \\
\hline $\mathrm{CM}(\mathrm{mm})^{1}$ & $53,33 \pm 4,49$ & $52,94 \pm 5,87$ \\
\hline $\operatorname{MinMic}(\mu)^{1}$ & $21,88 \pm 1,43$ & $21,96 \pm 1,43$ \\
\hline $\operatorname{MaxMic}(\mu)^{1}$ & $27,53 \pm 1,57$ & $28,02 \pm 2,14$ \\
\hline $\operatorname{DistPF}(\mathrm{mm})^{1}$ & $46,33 \pm 6,11$ & $44,11 \pm 5,37$ \\
\hline $\operatorname{CVR}(\% / \mathrm{mm})^{1}$ & $56,72 \pm 8,36$ & $55,54 \pm 5,59$ \\
\hline $\begin{array}{l}{ }^{1} \text { Médias seguida } \\
{ }^{2} \text { Médias seguida } \\
\mathrm{CL}=\text { crescimentc } \\
\text { de ondulações } \\
\text { longo da mech } \\
\text { representa apro } \\
\text { coeficiente de v } \\
\text { fibras mais gros } \\
\text { seja, porcentag } \\
\text { diâmetro da fib } \\
\text { micras; } \mathrm{CM}=\text { co } \\
\text { MaxMic= ponto }\end{array}$ & $\begin{array}{l}\text { ha, diferem estatistic } \\
\text { hha, diferem estatistic } \\
\text { de fevereiro até ago } \\
\text { da fibra de lã em m } \\
\text { escimento em micras } \\
\text { ras que discrepam d } \\
=\text { o número de micras } \\
\text { ntagem de fibras me } \\
\text { igual a } 30 \text { micras; } \\
\text { ão do diâmetro, repr } \\
\text { milímetro; MinMic= } \\
\text { eecha em micras; Dist }\end{array}$ & $\begin{array}{l}\text { e de Fisher }(p<0,05) \text {. } \\
\text { e de Kruskal-Wallis ( } p<0,05) \text {. } \\
\text { deçãa; Ondo do diâmetro da fibra ao } \\
\text { ião estatística em micras que } \\
\text { o das fibras da amostra; } C V D= \\
\text { do diâmetro médio de } 5 \% \text { das } \\
\text { ras; } F C=\text { fator de conforto, ou } \\
\text { que leva em consideração o } \\
\text { idade do fiado expressado em } \\
\text { o longo da mecha em micras; } \\
\text { oonta até o ponto mais fino ao }\end{array}$ \\
\hline
\end{tabular}

Existe variação da resistência entre animais da mesma raça, assim como entre raças diferentes (STEAR; MURRAY, 1994). Amarante et al. (2004), observaram que cordeiros das raças Santa Inês e Suffolk, mantidos sob as mesmas condições de manejo até 12-14 meses de idade, apresentaram diferenças em relação à susceptibilidade aos nematoides parasitas, pois $70 \%$ dos cordeiros Santa Inês mostraram-se resistentes, enquanto que $80 \%$ dos 
cordeiros Suffolk mostraram-se suscetíveis. De modo semelhante, Rocha et al. (2005) demonstraram que cordeiros Santa Inês, antes da desmama, são mais resistentes às infecções naturais por nematoides gastrintestinais do que cordeiros lle de France. A maior resistência aos helmintos observada em raças menos produtivas, tanto de carne como de lã, não tem sido de grande utilização em programas de cruzamento comercial, pelo fato de não atingirem os mesmos níveis produtivos que aquelas especializadas (BENAVIDES, 2009), entretanto, na atualidade, com os elevados níveis de resistência dos helmintos aos vermífugos, as características de resistência e resiliência demonstradas por essas raças menos especializadas são uma reserva genética para ser utilizada.

\section{CONCLUSÃO}

Nas condições em que foi desenvolvido este estudo, à exceção do ganho em comprimento, não foram verificadas diferenças produtivas significativas entre os ovinos com diferentes níveis de infecção parasitária. Contudo, a seleção de animais resistentes aos helmintos parasitas apresenta-se como uma importante ferramenta para obter-se níveis aceitáveis de produtividade. 


\title{
PRODUCTIVITY OF CORRIEDALE LAMBS PHENOTYPICALLY RESISTANT TO GASTROINTESTINAL NEMATODES
}

\begin{abstract}
The search for nematode-resistant sheep is a trend within productive systems, but productivity must be taken into account, because selecting animals resistant to parasitic action worths nothing if they do not meet the production parameters required by the market. The objective of this study was to quantify the productive characteristics related to weight gain, growth of body components, body score, body conformation and quality of wool, relating them to the egg count per gram of feces (FEC), characterizing a likely individual resistance expression to these nematodes within a year of life. The experiment was conducted in the city of Herval (RS). 32 Corriedale lambs were used, they were evaluated from three to 12 months of age. At the end of the experiment they were classified into two groups, low infection (EPG average of each animal below 900 throughout the trial) and high infection (EPG average of each animal above 1000 throughout the trial). With the exception of the body growth parameter $(p<0.05)$ for the other variables studied no significant differences were found among the animals with different levels of parasitic infection.
\end{abstract}

Keywords: Sheep. Productive Indexes. Worms.

\section{PRODUCTIVIDAD DE CORDERAS CORRIEDALE FENOTIPICAMENTE RESISTENTES A NEMATODOS GASTROINTESTINALES}

\section{RESUMEN}

a búsqueda de ovejas inmunes a la acción de nematodos es una tendencia dentro de los sistemas productivos, todavía se debe tomar en cuenta la productividad, pero de - poco vale la selección de animales resistentes a la acción del parásito, si no alcanza índices de producción compatibles con la presión que requiere el mercado. Así que el objetivo del presente estudio fue cuantificar el aumento de las características productivas relacionadas con peso, crecimiento de componentes del cuerpo, acabamiento, conformación y de calidad de la lana en relación con el recuento de huevos por gramo de heces, caracterizando así una probable expresión individual de resistencia a estos nematodos a lo largo de un año de vida. El ensayo fue realizado en el municipio de Herval (RS). Se utilizaron 32 corderas de la raza Corriedale, siendo evaluadas de los tres hasta los 12 meses de edad. Al final del ensayo fueron clasificadas en dos grupos, infección baja (HPG promedio de cada animal debajo de los 900 a lo largo del ensayo) e infección elevada (HPG promedio de cada animal encima de los 1000 a lo largo del ensayo). A excepción del 
parámetro de crecimiento del largo del cuerpo $(p<0,05)$, para las demás variables estudiadas, no fueron encontradas diferencias significativas entre los animales con distintos niveles de infección por parásitos.

Palabras clave: Ovinos. Índices productivos. Parasitósis.

\section{REFERÊNCIAS}

AMARANTE, A. F. T.; BRICARELLO, P. A.; ROCHA, R. A.; et al. Resistance of Santa Ines, Suffolk and lle de France lambs to naturally acquired gastrointestinal nematode infections.

Veterinary Parasitology, v. 120, p. 91-106, 2004.

AMARANTE, A. F. T. Resistência genética a helmintos gastrointestinais. In: SIMPÓSIO DA SOCIEDADE BRASILEIRA DE MELHORAMENTO ANIMAL, 5, 2004, Pirassununga. Anais. São Paulo: SBMA, 2004.

AMARANTE, A. F. T. Nematoides gastrintestinais em ovinos. In: CAVALCANTE, A. C. R.; VIEIRA, L. S.; CHAGAS, A. C. S.; MOLENTO, M. B. (Eds.). Doenças parasitárias de caprinos e ovinos: epidemiologia e controle. Brasília: Embrapa Informação Tecnológica, p. 17-62, 2009.

BALIC, A.; BOWLES, V. M.; MEEUSEN, E. N. T. The immunobiology of gastrointestinal nematode infections in ruminants. Advances in Parasitology, v. 45, p. 181-241, 2000.

BENAVIDES, M. V. Prós e contras da resistência genética dos ovinos aos helmintos gastrintestinais. Documentos, Bagé, Rio Grande do Sul. Documentos, EMBRAPA ISSN 19825390, v. 79, 2008.

BENAVIDES, M. V. Marcadores moleculares para resistência. In: CAVALCANTE, A. C. R.; VIEIRA, L. S.; CHAGAS, A. C. S.; MOLENTO, M. B. (Eds.). Doenças parasitárias de caprinos e ovinos: epidemiologia e controle. Brasília: Embrapa Informação Tecnológica, p. 508-548, 2009.

BENAVIDES, M. V.; SOUZA, C. J. H.; MORAES, J. C. F.; BERNE, M. F. A.; LEDUR, M. C. Resistência a parasitos gastrintestinais de ovinos Merino Australiano no RS e suas correlações com características produtivas. In: SIMPÓSIO IBEROAMERICANO SOBRE CONSERVACIÓN Y UTILIZACIÓN DE RECURSOS ZOOGENÉTICOS, 11, 2010, João Pessoa. Anais. Paraíba, 2010.

BISHOP, S. C.; STEAR, M. J. Genetic and epidemiological relationships between productivity and disease resistance: gastro-intestinal parasite infection in growing lambs. Animal Science, v. 69, p. 515-524, 1999.

BUENO, M. S.; CUNHA. E. A.; SANTOS, L. E.; et al. Sistema intensivo de produção de ovinos para abate: controle da verminose. In: SIMPÓSIO SOBRE CONTROLE DE PARASITAS EM 
PEQUENOS RUMINANTES, 2005, São Paulo. Anais. Nova Odessa: Instituto de Zootecnia, 2005, p. 100.

BUZZULINI, C.; SILVA SOBRINHO, A. G.; COSTA, A. J.; et al. Eficácia anti-helmíntica comparativa da associação albendazole, levamisole e ivermectina à moxidectina em ovinos. Pesquisa Agropecuária Brasileira, Brasília, v. 42, n. 6, p. 891-895, 2007.

CABRAL, L. S.; NEVES, E. M. O.; ZERVOUDAKIS, J. T.; et al. Estimativas dos requisitos nutricionais de ovinos em condições brasileiras. Revista Brasileira de Saúde e Produção Animal, v. 9, n. 3, p. 529-542, 2008.

COSTA, V. M. M.; SIMÕES, S. V. D; RIET-CORREA, F. Controle das parasitoses gastrintestinais em ovinos e caprinos na região semiárida do Nordeste do Brasil. Pesquisa Veterinária Brasileira, v. 31, n. 1, 2011.

EADY, S. J.; WOOLASTON, R. R.; LEWER, R. P.; et al. Resistance to nematode parasites in Merino sheep: correlation with production traits. Australian Journal of Agricultural Research, v. 49, p. 1201-1211, 1998.

FERNANDES, S. R. Perfis bioquímicos, hematológicos e características de carcaça de cordeiros em diferentes sistemas de terminação. Curitiba: UFPR, 2010. 59p. Dissertação (Mestrado em Ciências Veterinárias), Departamento de Zootecnia, Setor de Ciências Agrárias Universidade Federal do Paraná, 2010.

GORDON, H. McL.; WHITLOCK, H. V. A new technique for counting nematode eggs in sheep faeces. Journal of the Council for Scientific and Industrial Research, v. 12, p. 50, 1939.

MCCLURE, S. J. Sheep immunity to gastrointestinal nematode parasites-review 2000. CSIRO Livestock Industries, F. D. McMaster Laboratory. Armidale, Australia. http://www.csiro.au/scips, 2000.

OSÓRIO, J. C. S.; OSÓRIO, M. T. M. Produção de carne ovina: técnicas de avaliação in vivo e na carcaça. Programa de Pós-Graduação em Zootecnia, Universidade Federal de Pelotas, 2003. 73p.

OSÓRIO, J. C. S.; OSÓRIO, M. T. M.; FERNANDES, A. R. M.; et al. Técnicas de Avaliação In Vivo, na Carcaça e na Carne. IN: SELAIVE, A. B.; OSÓRIO, J. C. S. Produção de Ovinos no Brasil. Cap. 33, p. 527-550, 2014.

PINTO DE ANDRADE, L.; RODRIGUES, A. M.; VARZEA RODRIGUES, J.; et al. Caracterização da qualidade da fibra lanar de um efectivo Merino Branco. In: Jornadas “Ovelhas de Raça Merina", Sociedade Portuguesa de Ovinotecnia e Caprinotecnia, Porto Alegre, p. 8, 1999. 
ROCHA, R. A.; AMARANTE, A. F. T.; BRICARELLO, P. A. Resistance of Santa Inês and lle de France suckling lambs to gastrointestinal nematode infections. Revista Brasileira de Parasitologia Veterinária, v. 14, p. 17-20, 2005.

SALGADO, J. A. Sistemas De Produção De Cordeiros E Seu Efeito Na Infecção por Helmintos Gastrintestinais. Curitiba: UFPR, 2011. 66p. Dissertação (Mestrado em Ciências Veterinárias), Departamento de Zootecnia, Setor de Ciências Agrárias, Universidade Federal do Paraná, 2011.

SCHICHOWSKI, C.; MOORS, E.; GAULY, M. Influence of weaning age and an experimental Haemonchus contortus infection on behaviour and growth rates of lambs. Applied Animal Behaviour Science, v. 125, p. 103-108, 2010.

SILVEIRA, F. A.; FERREIRA, O. G. L.; FARIAS, P. P.; et al. Teste de eficiência de anti-helmínticos em ovinos sob sistema de manejo semi-extensivo. In: CONGRESSO BRASILEIRO DE ZOOTECNIA, 23, 2013, Foz do Iguaçu. Anais. Foz do Iguaçu: SBZ, 2013.

STEAR, M. J.; MURRAY, M. Genetic resistance to parasitic disease: particularly of resistance in ruminants to gastrointestinal nematodes. Veterinary Parasitology, v. 54, p. 161-176, 1994.

UENO, H.; GONÇALVES, P. C. Manual para Diagnóstico das Helmintoses de Ruminantes. 4. ed. Japan International Cooperation Agency. 1998. 143p.

WILLIAMSON, J. F.; BLAIR, H. T.; GARRICK, D. J.; et al. Parasitism and production in fleeceweight-selected and control sheep. New Zealand Journal of Agricultural Research, v. 38, p. 381-387, 1995a.

WILLIAMSON, J. F.; BLAIR, H. T.; GARRICK, D. J.; et al. Parasitological characteristics of fleeceweight-selected and control sheep. New Zealand Journal of Agricultural Research, v. 38, p. 389-397, 1995b.

WOOLASTON, R. R.; BAKER, R. L. Prospects of breeding small ruminants for resistance to internal parasites. International Journal for Parasitology, Oxford, v. 22, p. 377-380, 1992.

Autor para correspondência:

Fernando Amarilho-Silveira.

Universidade Federal de Pelotas, Faculdade de Agronomia Eliseu Maciel, Campus Universitário, Capão do Leão

(RS), Brasil, CEP 96010-900.

amarillo@zootecnista.com.br 